\title{
Design and realization of a data recorder
}

\author{
Jing Zhao \\ The First Aviation College of Air Force, Aviation Electronic Department, Xinyang, \\ Henan Province China \\ E-mail: sherilljames@.163.com
}

\begin{abstract}
USB interface technology is more and more popular. It is applied into hardware design profiting from its advantages. It has high speed and convenience. This article introduces the design and implementation methods of a kind of airborne data recorder. It emphasizes the application of USB interface technology into this system design and takes focus on the key problems, such as chip selection, firmware design and driver program.

Keywords: USB; Firmware; Driver Program
\end{abstract}

\section{Introduction}

Compared with a serial port or parallel port, USB has high speed and is convenient. It reduces troubles caused by hardware configuration and computer on-off because it can support hot plug in and out. This interface is the real one can realize when it inserts into a system, it can work. The USB interface speed is very high. The full speed is $12 \mathrm{M}$ bps according to the USB1.1 protocol. A serial port, its transmission speed is $115 \mathrm{k}$ bps; the speed of a parallel port is $1 \mathrm{M} \mathrm{bps.}$ So, USB is faster than 100 times of a serial port and 10 times of a parallel port. Based on these reasons, USB is more and more popular in hardware design region.

This data recorder is designed for an airborne device. It can implement real time data recording and computer communications. The communication part is realized through a USB port. After finishing data recording, the recorder stores data into a computer through a USB port controlled by client end software explored in VC++ environment. It is very clear the USB port is a certain port for transmitting data. Its working efficiency can influence the performance of this system. Following content will introduce how to design the USB port in this system. 


\section{USB Hardware Design}

\subsection{Hardware Structure}

The first step of a USB port design is to choose a USB chip according to the function requirements of a system and the port's status. From controlling type, USB chips can be divided into two categories. The first type includes a controller, and the second type needs an external controller. That is to say, the first type integrates a microchip or other controllers into its chip. A designer can program a firmware program and data disporting program depending on a programming environment and language according to different controllers. To the other type, a USB chip is only a serial port engine. It must communicate with an external controller by an interruption connection. A designer programs into the external controller. In all, a PDIUSBD12 (abbreviation to D12) chip is applied in this system.

\subsubsection{Basic information of D12}

D12 is a USB chip having perfect performance. It needs an external controller to implement USB device enumeration and data disporting. D12 has a serial interface engine, a 32k bits FIFO storage, a transceiver, a voltage adjuster, a programmable clock output, a controllable soft connection, an inner power on reset circuit and a low power reset circuit. It allows local DMA (direct memory access) transmission. Its theory block diagram is shown on figure 1.

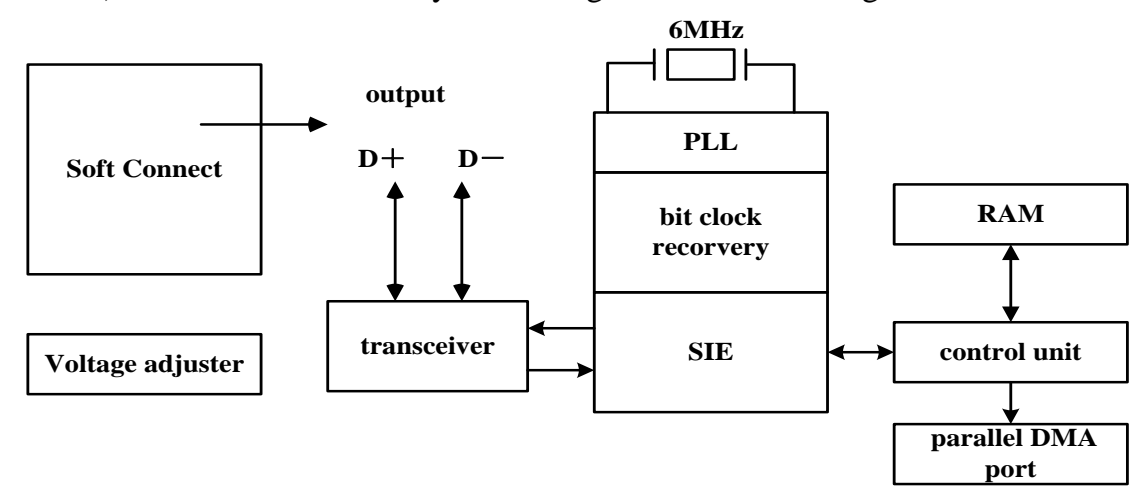

Fig. 1 D12 construction block diagram

Almost all of D12 chip's capsulations have 28 pins. Includes 8-bit data line (Data0 Data7), 1 bit address line to distinguish write and read commanders, a 
low level available interruption output pin, read enable pin and a write enable pin.

D12 obeys USB 1.1 protocol. It supports three endpoints. Each endpoint has its own transmitting and receiving buffers. There are a control endpoint, a main endpoint and a normal endpoint. The main endpoint is configured double buffers to increase throughput and realize real time transmission. Under block transmission mode, the endpoint's speed is $1 \mathrm{M}$ bps. There is a GoodLink connection lamb blinking to show data transmission, which can convenient a debugging process.

\subsubsection{Hardware Connection}

The following figure is the hardware structure of this data recorder. It has a FPGA, a FLASH data accumulator, a 485 interface chip, a D12 chip and a DSP. The DSP chip is the external controller of D12.

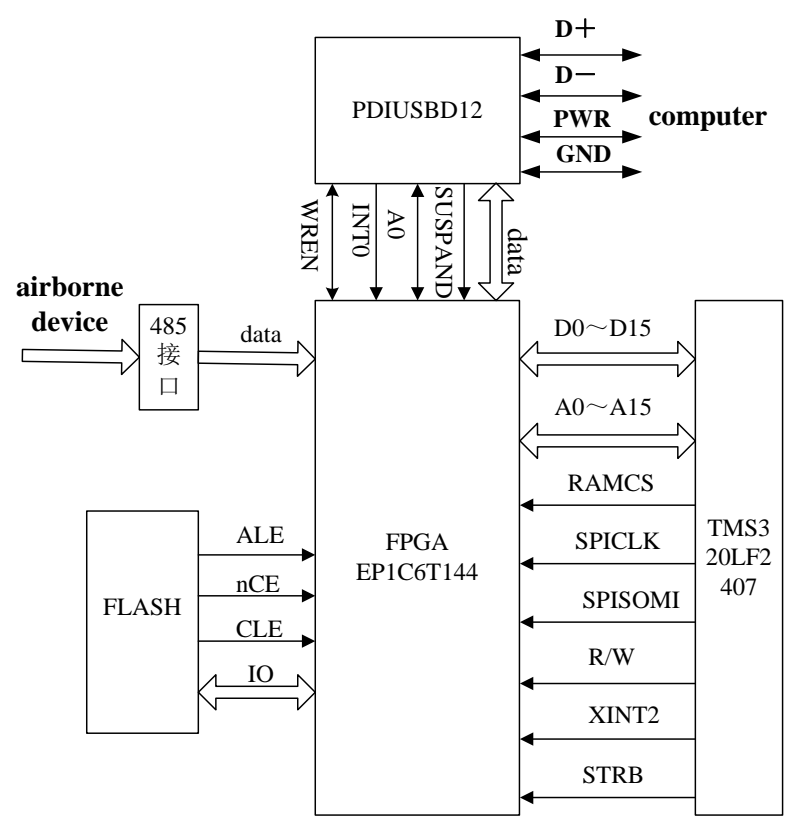

Fig. 2 Hardware Connection Figure

To this figure, the D12 has two connection parts. The first one is a computer, as a master device; the second is a FPGA chip. 
To the computer, this data recorder is a USB slave device, which means any communications would be started from this computer. Any information this computer is required, this recorder replies. A USB interface connects to this computer by four lines. There are VCC (power line), GND (ground line), D+ and D- (differential data line). The computer decides the status of slave devices by detecting the voltage changes of data lines. When there is no device connecting, two lines reach ground by two pull down resistances. When any data lines reaches to VCC level, it means the slave device is ready. When D+ is high means the device is a full speed device, otherwise, this is a low speed device. The D12 chip can realize full speed recognition by connecting a $1.5 \mathrm{~K}$ pull up resistance.

To the USB device, this FPGA chip does not control anything except for connecting some key lines to a DSP chip. This DSP chip is TMS320LF2407. This chip is a classic type of TI Company. This chip applies Harvard structure, $3.3 \mathrm{v}$ power supplying, 4-stage stream line, 40MIPS executing speed, 20ns instruction cycle. It has $32 \mathrm{~K}$ bytes flash program storage, $2.5 \mathrm{~K}$ bytes inner RAM, two events administrators, 16-channel 10-bit AD converter, a CAN, a SPI and a SCI many inner modes. The D12 chip connects this DSP with 8-bit data bus, A0 address line and an interruption line.

\subsection{Firmware Program}

A USB device has four transmission modes: control transmission, bulk transmission, interruption transmission and synchronization transmission. The control transmission is to enumerate a USB device. When a device connects a computer, the computer sends a setup packet though endpoint 0 , which includes standard USB requirements. The D12 chip generates an interruption signal to the controller, DSP. The DSP replies each requirement by accessing the interruption register and the last transmission status register, and sends back information by the same endpoint. The computer reads the data to get description information to allocate and load a device driver program and configures this device. After this, this device can transmit data by endpoints which are available to the configuration. The data transmits in bulk. The maximum data of each transmission is 64 bytes. If the data is larger than 64 bytes, it transmits in batch. The following figure is the main program flow of the firmware of D12 chip and data disposing program. 


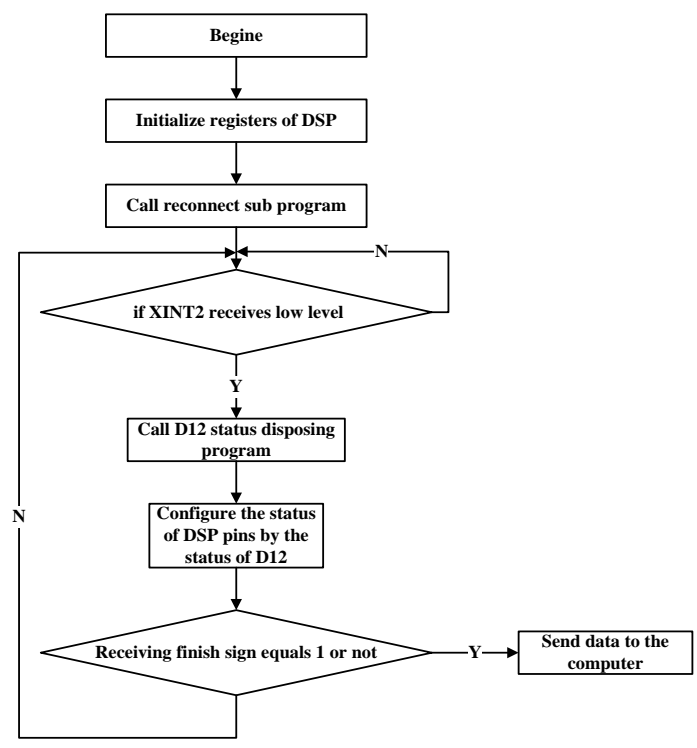

Fig. 3 Firmware Program Flow Figure

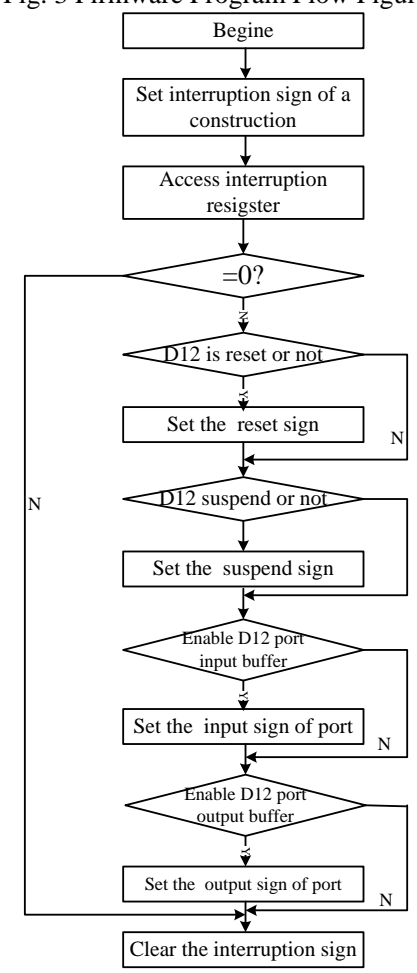

Fig. 4 PDIUSBD12 status processing program flow 
There are several problems should be paid attention to by applying 2407 as the controller of D12 chip: the first is the configuration information is stored into 2407 as its inner format; the second is the design of .cmd document for 2407 program and data configuration; the third is the setup of dual level interruption construct and interruption vector because there are many interruption sources in 2407; the last one is the external interruption could not triggered by level but edge, the interruption for D 12 is level available, and this problem can be solved by FPGA.

\section{Driven Program and Application Program}

The driver program of USB device is based on WDM. Under Windows exploration environment, the customer can build the communication between application software to hardware by a driving development tool. Among these development tools, the Windriver developed by Jungo Company has high quality of its efficiency and good compatibility with no relationship with system program. The products provided by Windriver are API functions by enveloping all complicate operations. The driving program could be done with a little change according to the projects. By this way, not only the labor of developers could be simplified, but also the developing time has been saved.

The application software is developed by $\mathrm{VC}++$, which includes communication dynamic link library (DLL) program with driver program and control program. DLL is an executable module enveloped some functions to upload storage space from USB interface buffer to PC memory. Then, add the DLL into communication program to initialize, write and read USB device, and data reading, erasing and control from recorder.

\section{Conclusion}

Applying USB port in a data recorder, not only solve the problems of installation, but also provide high speed data transmission. It has been proved in practice that this recorder can satisfied the requirements of contractor, and shows high quality in implementation.

\section{References}

1. Chendehuang, "A Design of a Control and Test Platform of a Synthesis Communication System”, Computer Engineering, PP. 225-227.2005. 
2. Wushudong and Nietao, "Design of Aviation Communication Eequipment Automated Test Instrument", Missile and Guidance Report, PP.307310.2006.

3. Wanglei and Fanglian, "The Analog and Digital Signal Source Design Based on FPGA", Science and Technology Consulting. PP. 30-31.2012

4. Xufuxin, "Design of Test System Based on Wireless Connection", Computer Engineering, PP.84-89.2012. 